\title{
Hypofractionation not associated with reduced toxicity
}

Hypofractionated radiotherapy is not noninferior to conventional radiotherapy in terms of acute gastrointestinal and genitourinary adverse effects in men with intermediate-risk and high-risk prostate cancer, say investigators involved in the HYPRO trial.

This phase III randomized trial was designed to investigate the effect of hypofractionation on relapse-free survival in patients with prostate cancer. Aluwini et al. randomly assigned 820 men with intermediate-risk and high-risk prostate cancer at seven radiotherapy centres in the Netherlands to receive standard

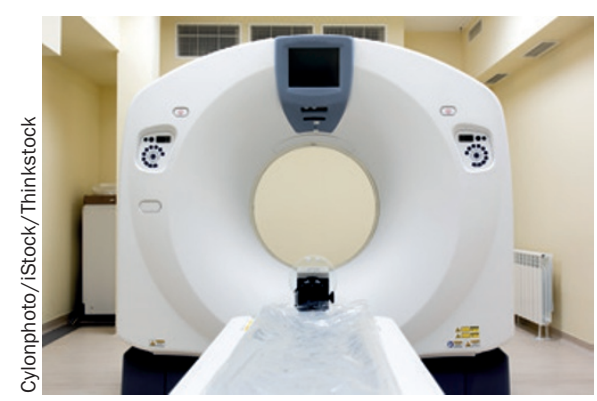

fractionation ( 39 fractions of $2 \mathrm{~Gy}$ in 8 weeks; $n=410$ ) or hypofractionation ( 19 fractions of $3.4 \mathrm{~Gy}$ in 6.5 weeks; $n=410$ ).

The investigators found that 3 months after radiotherapy, the prevalence of grade 2 or worse gastrointestinal toxicity was similar in the hypofractionation and standard fractionation groups (23\% versus $22 \%$ ); they also found that 3 months after radiotherapy, the prevalence of grade 2 or worse genitourinary toxicity was similar in the two groups (13\% in each group).

Aluwini and co-workers found that there was no significant difference in the cumulative incidence of grade 2 or worse acute genitourinary toxicity up to 120 days after radiotherapy between the hypofractionation and standard fractionation groups (60.5\% versus $57.8 \%$; $P=0.43)$. They did, however, find that the cumulative incidence of grade 2 or worse acute gastrointestinal toxicity up to 120 days after radiotherapy was significantly higher in the hypofractionation group than in the standard fractionation group (42.0\% versus $31.2 \% ; P=0.0015)$.
"Although 3 months after completion of radiotherapy this difference had dissipated," say the authors, "it is possible that late gastrointestinal toxicity will be worse, due to the known association between acute and late toxicity; patients remain in follow-up and we will investigate this association in the future."

The researchers also found that the presence of baseline grade 2 or worse genitourinary or gastrointestinal symptoms were associated with increased cumulative incidences of acute genitourinary or gastrointestinal toxicities, respectively, in both groups. In addition, they report that the risk of acute gastrointestinal toxicity decreased with the use of neoadjuvant hormonal therapy.

Rebecca Kelsey

Original article Aluwini, S. et al. Hypofractionated versus conventionally fractionated radiotherapy for patients with prostate cancer (HYPRO): acute toxicity results from a randomised non-inferiority phase 3 trial. Lancet Oncol. doi:10.1016/S1470-2045(14)70482-6 